\title{
¿Podemos predecir clínicamente la cervicoartrosis?
}

\author{
F. Borrell-Carrió, A. Bossy Azpilicueta ${ }^{1}$, D. Herrero Barrera ${ }^{2}$, M. Colás \\ TAUGi², R. López Pisa ${ }^{3}$, C. VAlero García ${ }^{4}$ \\ Especialista en Medicina Familiar y Comunitaria. Doctor en Medicina. Universidad \\ de Barcelona. ABS Gavarra. DAP Baix Llobregat Centre. ICS. 'Especialista en \\ Radiodiagnóstico. Servei de Radiodiagnòstic. DAP Baix Llobregat Centre. ICS. ${ }^{2}$ Espe- \\ cialista en Medicina Familiar y Comunitaria. Universidad de Barcelona. ABS Gava- \\ rra. DAP Baix Llobregat Centre. ICS. 'Diplomada en Enfermería. ABS Gavarra. \\ DAP Baix Llobregat Centre. ICS. ${ }^{4}$ Especialista en Salut Pública. \\ DAP Baix Llobregat Centre. ICS. Barcelona
}

\section{RESUMEN}

Fundamento: desconocimiento del valor de la exploración física cervical.

Objetivo: establecer la utilidad y predictividad de algunas maniobras cervicales en relación a la presencia de cervicoartrosis.

Diseño: estudio descriptivo transversal con muestreo sistemático.

Pacientes y métodos: sobre una muestra de 100 personas que se realizaban una exploración radiológica cervical en el Servicio de Radiodiagnóstico de la DAP Cornellà Centre (enero a mayo 2000, muestreo sistemático), evaluamos para cada una de las personas dos pruebas de exploración física (test de rotación cervical medida con goniómetro estático y test barbilla-pecho), comparando esta evaluación con la obtenida en la exploración radiológica, efectuada por un radiólogo que desconocía el resultado de la exploración clínica.

Resultados: cuando la rotación cervical lateral era menor de $56^{\circ}$, obteníamos un valor predictivo positivo del $85 \%$, una sensibilidad del $81 \%$ y una especificidad del 61\%. La valoración clínica cervical con estos dos tests se correlacionaba de forma significativa con la gravedad radiológica, sin llegar a hacerlo con la concordancia (índice Kappa). Una maniobra de rotación cervical normal (igual o mayor a $70^{\circ}$ ) excluía la presencia de cervicoartrosis.

Conclusiones: el test de rotación cervical tiene relevancia clínica y la combinación con otras maniobras exploratorias permite un cribado clínico previo y complementario a la evaluación radiológica.

Can we predict cervicoarthrosis clinically?

\section{ABSTRACT}

Background: ignorance of the value of the physical cervical exploration.

Objective: to establish the predictivity and utility of several cervical maneuvres in relation to cervicoarthrosis.

Design: descriptive transversal study with systematic sampling.

Patients and methods: a series of 100 patients whom were realized a cervical radiological exploration in the Radiodiagnosis Service (from January to March 2000), were evaluated with two physical explorations (cervical rotation test measured with a static goniometer and chest-chin test). The results were compared with the evaluation we obtain of the radiological exploration that was realized by a radiologists who did not know the result of the clinical exploration.

Results: when the lateral cervical rotation was smaller than 56 degrees, we obtained a positive predictive value of $85 \%$, a sensibility of $81 \%$ and a specificity of $61 \%$. The cervical clinical assesment with these two tests was correlated in a significant way with the radiological examination, without achieving a significant Kappa index. A maneuver of normal cervical rotation (same or bigger than 70 degrees) excluded cervicoartrosis.

Conclusions: the cervical rotation test has a clinical relevance and the combination with other exploratory maneouvres act as a clinical tool for the radiological evaluation. 
Palabras clave: Cervicoatrosis. Exploración fisica. Exploración radiológica.
Key words: Cervicoarthrosis. Physicial diagnosis. X-ray diagnosis.

\section{INTRODUCCIÓN}

El dolor cervical es un motivo frecuente de consulta en el ámbito de Atención Primaria: un tercio de la población lo experimentará a lo largo de su $v_{\text {vida }}$. El médico de familia frecuentemente tendrá que responder a la pregunta de sus pacientes: ¿tengo artrosis cervical? La exploración física de la columna cervical se ha visto desplazada por la exploración radiológica cuando, de hecho, ocupa menos de 3 minutos de nuestra consulta y, desde nuestra experiencia, aporta información relevante y complementaria. Estudios anteriores han comparado la información obtenida por la mielografía y otras técnicas radiológicas con la limitación funcional y la sintomatología compresiva medular ${ }^{2-5}$, pero no se ha delimitado el valor de las maniobras exploratorias de la columna cervical, su sensibilidad, especificidad y valor predictivo en relación a la patología artrósica. El presente trabajo parte de nuestras observaciones clínicas cotidianas, según la cual una rotación cervical normal casi nunca se asocia de una cervicoartrosis radiológica significativa, para contestar así a las siguientes preguntas:

1. ¿Qué aporta la maniobra de rotación lateral de la columna cervical a la hora de determinar si un paciente tiene cervicoartrosis?

2. Cuando la maniobra de rotación lateral es normal, ¿tenemos algún peligro de que nos encontremos delante de un paciente con cervicoartrosis?

3. Cuando la maniobra está claramente limitada, ¿cuál es el riesgo de que sospechemos cervicoartrosis sin que el paciente la tenga?

\section{METODOLOGÍA}

Tipo de estudio: transversal con muestreo sistemático.

Definición de caso y duración: durante los meses de enero a mayo del año 2000, evaluamos a todos los pacientes que se realizaban una radiografía cervical en el Servicio de Radiodiagnóstico de la SAP Baix Llobregat Centre según el protocolo de recogida de datos, independientemente de cual fuera el motivo de la radiografía.

Criterios de exclusión: excluímos a los pacientes que presentaban tortícolis, cervicalgia aguda y síndrome del latigazo cervical, ya que en éstos el componente de contractura muscular es muy alto.

Protocolo de estudio: el protocolo de estudio constaba de dos partes:

1. En la parte clínica, un único observador obtuvo los datos personales, motivo de consulta, consumo de analgésicos, tipo de trabajo habitual y exploración de la columna cervical. Dicha exploración consistía en la medida del grado de las rotaciones cervicales mediante el goniómetro estático y la maniobra barbilla-pecho, constatando la presencia/ausencia de dolor al realizar estas maniobras.

Graduar correctamente las maniobras de rotación cervical constituyó la piedra angular del trabajo. Para valorarla, seguimos la sistemática propuesta por Seidel ${ }^{6}$, haciendo servir un aparato denominado goniómetro estático. Situamos un goniómetro de $50 \mathrm{~cm}$ en el saliente móvil de un tallímetro (Fig. 1), de manera que su generatriz coincidiera con el punto de giro de la cabeza. El paciente se colocaba unas gafas con un puntero (Fig. 2), que tenía que quedar justo por debajo del goniómetro. El ángulo de rotación se valoraba con la espalda totalmente paralela a la pared y realizando un giro hacia la derecha e izquierda (Fig. 3), señalando el puntero el ángulo conseguido. También se controlaba de forma visual cualquier rotación corporal por parte del paciente a la hora de hacer la rotación cervical para evitar que se generaran grados de rotación irreales.

La flexión cervical se valoró con la maniobra barbilla-pecho. En esta maniobra, el médico colocaba dos dedos (uno encima del otro) perpenciculares a la superficie del pecho del paciente y se le pe-

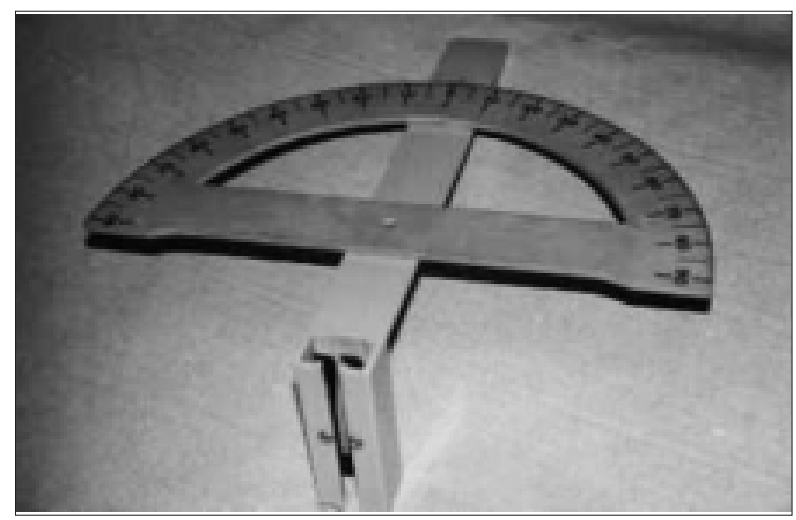

Figura 1

Goniómetro estático. 


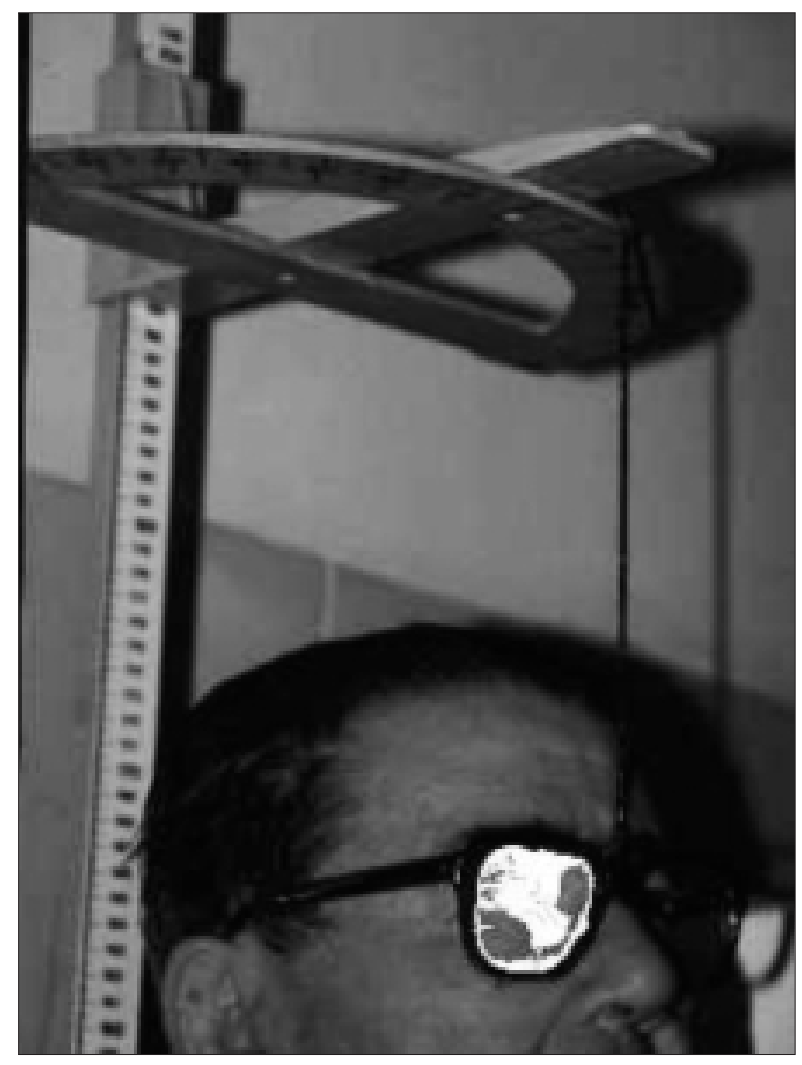

Figura 2 Posición cero grados.

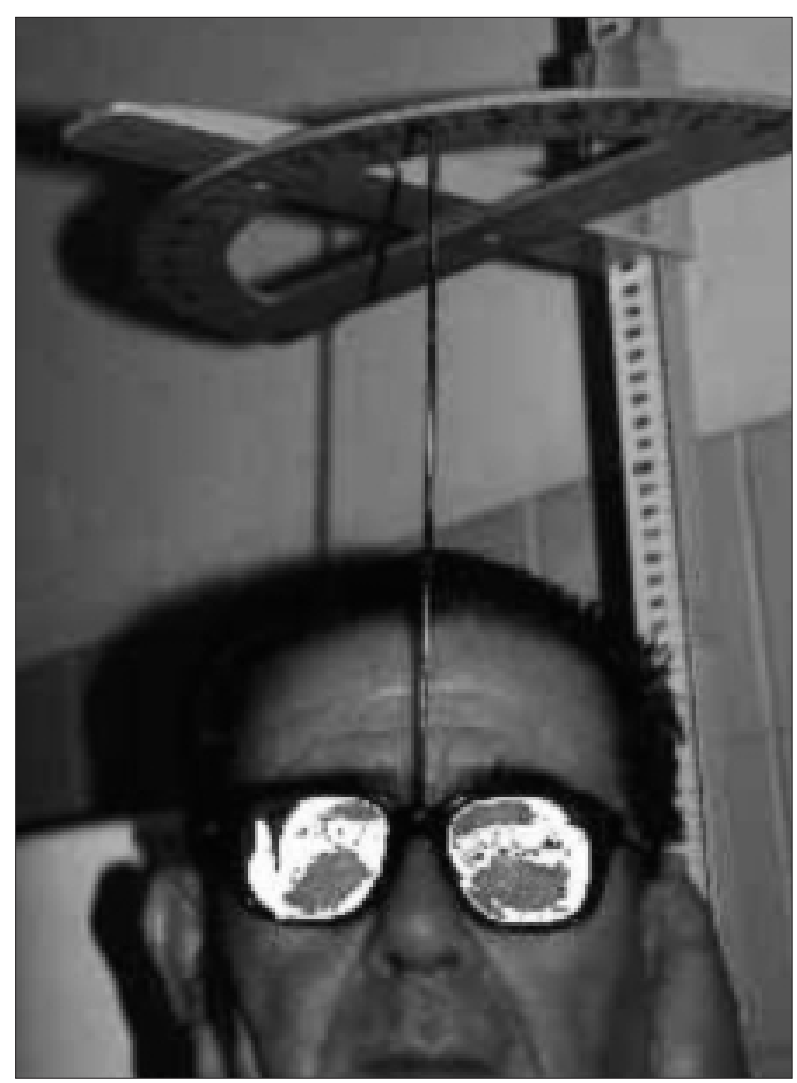

Figura 3

Rotación cervical izquierda. día que realizara una flexión máxima, con la intención de que tocara con la barbilla los dos dedos. Si era capaz de realizarlo, se consideraba normal, siendo esta variable únicamente cualitativa (sí/no).

2. La valoración radiológica fue realizada independientemente por un radiólogo experimentado que no conocía el resultado de la valoración clínica y, basándonos en los criterios de Resnick ${ }^{7}$, incluímos: a) presencia y tipo de pinzamiento articular (únicos-múltiples, anteriores-posteriores); b) presencia o no de esclerosis articular y/o inestabilidad articular; c) osteofitosis y su grado; y d) presencia de rectificación de la columna.

Definición de las variables: para las dos valoraciones, establecimos por consenso unas categorías de variables que quedan expresadas en las tablas I y II.

\section{Tabla I}

\section{CATEGORÍAS QUE EXPRESAN GRAVEDAD EN LA EXPLORACIÓN CLÍNICA}

1. Gravedad severa: media de las rotaciones laterales menor de 36 grados

2. Gravedad importante: media de las rotaciones laterales entre 36 a 55 grados con maniobra de flexión barbilla-pecho alterada

3. Gravedad moderada: media de las rotaciones laterales entre 36 a 55 grados con maniobra de flexión barbilla-pecho normal

4. Gravedad leve: media de las rotaciones laterales entre 56 a 65 grados

5. Normal: media de las rotaciones laterales igual o superior a 66 grados

\section{CATEGORÍAS QUE EXPRESAN GRAVEDAD EN LA EXPLORACIÓN RADIOLÓGICA}

1. Gravedad severa: pinzamientos múltiples y/o inestabilidad articular

2. Gravedad importante: pinzamiento único con presencia de osteofitosis grado III/IV, o pinzamiento único sin osteofitosis pero con la presencia de esclerosis

3. Gravedad moderada: pinzamiento único, o esclerosis, u osteofitosis grado III/IV

4. Gravedad leve: osteofitosis grado I/II

5. Normal: no presencia de signos radiológicos propios de cervicoartrosis

Nota: el concepto "pinzamiento" se refiere tanto a pinzamiento intersomático (afetación discal) como articular (unciformes y $/ 0$ apofisarias). 
Consideramos como gold standar la evaluación radiológica, aplicando el test de correlación de Pearson entre la evaluación radiológica y clínica, el análisis de concordancia Kappa, también entre ambas evaluaciones, y el análisis de la sensibilidad, especificidad, valor predictivo y razón de probabilidad del test (entendiendo por test la aplicación de la rotación cervical y "barbilla-pecho").

\section{RESULTADOS}

Descripción de la muestra: participaron en el estudio 106 pacientes provinientes fundamentalmente de Can Vidalet (30\%), La Gavarra (32\%), Luis Millet (6\%), Martí Julià (22\%) y Sant Just (8\%). Por edades 17 eran menores de 35 años, 20 de 36 a 45 años, 50 de 46 a 65 y 15 mayores de 65 años, siendo el $30 \%$ varones. Excluimos 6 casos por tener criterio de exclusión (cervicalgia aguda, tortícolis, latigazo). El 64\% de la muestra tomaba medicación antiálgica o antiinflamatoria y, de ellos, el $60 \%$ tenían que hacerlo diariamente. El 26\% de la muestra no tenía signos radiológicos de cervicoartrosis, de los cuales 1 fue clasificado a nivel de exploración física como de gravedad severa y 9 como de gravedad leve.

Variables en estudio: los casos considerados radiológicamente como severos, importantes y moderados sumaron 64 , de los cuales uno sólo fue considerado a nivel de exploración física como normal. La correlación de Pearson entre la valoración de la gravedad radiológica y la exploratoria fue de $0,4(p<0,0001)$. El test de concordancia Kappa fue de 0,05 (no significativo). En la tabla III expresamos las medidas relativas a sensibilidad, especificidad y valor predictivo, entre otros.

Tenemos que destacar que entre las personas que

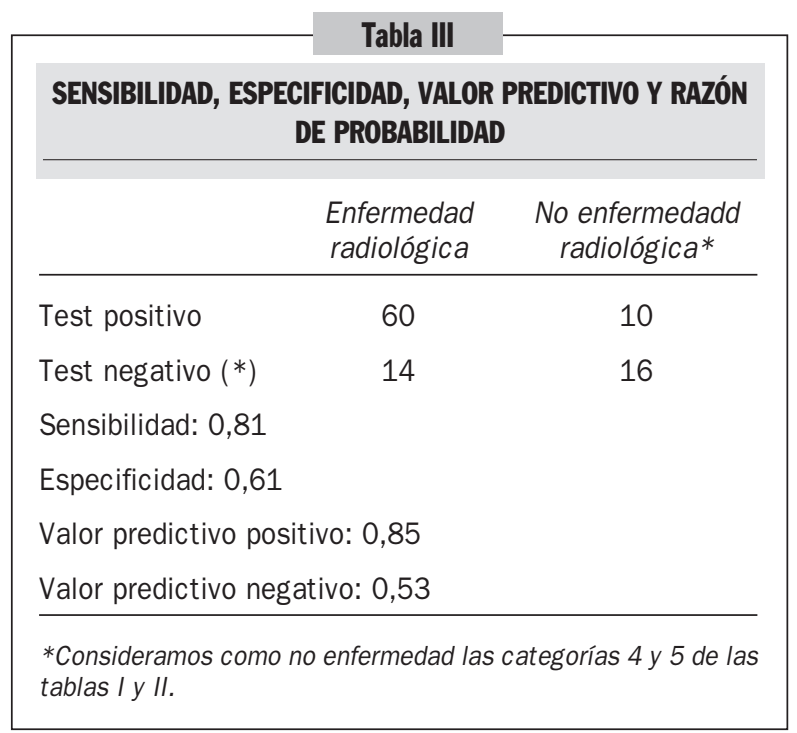

mostraron exploración igual o superior a 65 grados no existía ningún caso de cervicoartrosis. Entre las 9 personas que presentaban una exploración de rotación cervical inferior a 35 grados, todas presentaron cervicoartrosis importante, excepto una.

\section{DISCUSIÓN Y CONCLUSIONES}

Presentamos un método práctico para ponderar de manera sencilla la presencia de cervicoatrosis sin ayuda de exploraciones complementarias. A tal efecto, el clínico tiene que realizar la evaluación de las rotaciones laterales de la columna cervical y el test barbilla-pecho, exploraciones que casi no se realizan en la clínica diaria de manera suficientemente sistematizada, y que a su vez demuestran una correlación con la presencia radiológica de cervicoartrosis. He aquí la respuesta a las tres preguntas que nos formulábamos:

1. ¿Qué aporta la maniobra de rotación lateral de la columna cervical a la hora de determinar si un paciente tiene cervicoartrosis? Si consideramos esta prueba como test de cribado, obtenemos una buena sensibilidad $(81 \%)$ con una especificidad baja (61\%), perfil que corresponde a la mayor parte de maniobras de exploración física. El valor predictivo positivo es del $85 \%$, es decir, con la maniobra de rotación lateral cervical alterada (menor de 56 grados), ésta es la probabilidad de tener cervicoartrosis.

2. Cuando la maniobra de rotación lateral es normal, ¿tenemos algún peligro de que nos encontremos delante de un paciente con cervicoartrosis? La negatividad de esta exploración no excluye de manera suficiente la presencia de esta enfermedad (especificidad baja). La razón de probabilidad positiva nos indica que la persona con el test positivo tiene el doble de probabilidades que otra sin el test positivo de tener cervicoartrosis, y que si es negativo, sólo existe un tercio de dicha probabilidad (razón de probabilidad negativa). A destacar que la mitad de los casos que son subdiagnosticados por la maniobra exploratoria se deben a casos de pinzamiento único. A veces, estos pinzamientos tienen una repercusión dinámica leve, aún produciendo dolor. En este perfil de pacientes, al coger como criterio de valoración único la maniobra de rotación cervical, nos llevaría a infravalorar su patología, a pesar de que nos da información complementaria a la radiológica, de interés clínico per se.

Así pues la evaluación de la rotación cervical es un test con una buena sensibilidad, pero con una baja especificidad. Con una evaluación física normal (rotaciones normales), tenemos el 14\% de falsos negativos, es decir, un rendimiento similar al tránsito esófago-gastroduodenal en relación al ulcus duodenal (entre el 10 y el 15\%, según las series) y un per- 
fil similar al de les tiras reactivas de orina para la detección de piuria ${ }^{8}$. Otras pruebas tan famosas como la de McMurray y la de Apley, para la detección de meniscopatía, tienen una sensibilidad menor: del 45 y del $28 \%$ respectivamente ${ }^{9}$. Una maniobra tan reconocida como es la descompresión abdominal en el contexto de un abdomen agudo como sospecha de apendicitis, tiene una sensibilidad del $63 \%$ y una especificidad del $69 \%{ }^{10}$. La combinación de diferentes maniobras de exploración física mejora estos resultados. Así, por ejemplo, en el caso mencionado de la meniscopatía, resulta posible excluir su presencia con un 75 a $95 \%$ de probabilidad ${ }^{11,12}$ cuando combinamos 3 ó 4 maniobras, respectivamente. Por tanto, podemos decir que, en general, las maniobras físicas aisladas tienen rendimientos bajos, y que será justamente cuando las combinamos cuando obtenemos rendimientos altos, capaces de dar seguridad al clínico. La rotación cervical es una maniobra clínicamente relevante, pero otros estudios tendrán que profundizar en esta combinación de maniobras. Sí podemos avanzar que la maniobra de barbilla-pecho nos ha aportado poco, y que tenemos la impresión de que será justamente una maniobra que detecte las contracturas musculares la que podrá reducir los falsos positivos.

3. Cuando la maniobra está claramente limitada, ¿cuál es el riesgo de que sospechemos cervicoartrosis sin que el paciente la tenga?

La maniobra de rotación cervical es bastante orientativa, sobre todo cuando es claramente positiva o negativa; en estos casos extremos, la maniobra casi no tiene falsos negativos ni positivos.

Limitaciones del estudio. En la revisión de Medline desde 1975 que hemos realizado, no hemos encontrado ningún trabajo similar al nuestro. A destacar que el método descrito del goniómetro estático no está aún validado; esperamos tener la oportunidad de hacerlo en un futuro inmediato. Otra limita- ción del trabajo es que para usar el goniómetro el médico debe someterse a un mínimo entrenamiento. Otra limitación es el sesgo de la muestra, ya previamente seleccionada por los médicos como candidatos a hacerse una radiografia, y que, por tanto, ayuda a tener un valor predictivo más alto del que tendríamos en condiciones de consulta normal.

Finalmente entendemos que este trabajo es un buen exponente de lo que puede aportar una colaboración entre servicios clínicos y servicios de diagnóstico complementario, en este caso, radiodiagnóstico. También pone en evidencia la importancia de sistematizar y conocer los límites de las maniobras exploratorias clínicas que hemos heredado de la tradición médica, haciendo compatible su comprensión científica con el aspecto de arte que siempre conservarán. Sin duda, las habilidades clínicas en general y las de exploración física en concreto, constituyen un gran reto para la Medicina de Familia.

\section{AGRADECIMIENTOS}

A la inestimable ayuda del Centre de Documentació de 1'Institut Català de la Salut, Srta. M. Fernández Gironés. Al Dr. Josep Lluis Ibáñez, director SAP Baix Llobregat Centre, y al Dr. J. Pons Chalmeta, Jefe del Servicio de Radiodiagnóstico de la SAP, y a todos los miembros de dicho Servicio, por su apoyo al estudio.

\section{CORRESPONDENCIA:}

F. Borrell-Carrió

CAP Cornellà

C/ Bellaterra, 39, $2^{\text {a }}$ planta

08940 Cornellà de Llobregat, Barcelona

e-mail: fborrell.cp.ics@gencat.net

\section{Bibliografía}

1. Rojas M. Dolor cervical. En: SEMFyC Guía de actuación en Atención Primaria semFyC Barcelona, 1998.

2. Brooker AE, Barter RW. Cervical spondylosis. A clinica study with comparative radiology Brain 1965; 88 (5): 92536.

3. Truumees E, Herkowitz HN. Cervical spondylotic myelopathy and radiculopathy. Instr Course Lect 2000; 49: 33960 .

4. McClure P. The degenerative cervical spine: pathogenesis and rehabilitation concepts. J Hand Ther 2000; 13 (2): 163-74.

5. Young WF. Cervical spondylotic myelopathy: a common cause of spinal cord dysfunction in older persons. Am Fam Physician 2000; 62(5): 1064-70.

6. Seidel H, Ball J, Dains J, Benedict G. Manual Mosby de Exploración Física. $2^{a}$ ed. Madrid: Mosby, 1993.
7. Resnick. Diagnosis of bone and joint disorders. $3^{\mathrm{a}}$ ed. Philadelphia: WB Saunders Company, 1995.

8. Speicher CE. Elección de pruebas de laboratorio. Barcelona: Doyma, 1992. p. 123.

9. Kurosaka M, Yagi M, Yoshiya S, Muratsu H, Mizuno K. Efficacy of the axially loaded pivot shift test for the diagnosi of a meniscal tear Int Orthop 1999; 23 (5): 271-4.

10. Wagner J, McKinney P, Carpentenr JL. ¿Padece este paciente una apendicitis? JAMA (ed esp) 1997; 6 (4): 159-66.

11. Fowler PJ, Lubliner JA. The predictive value of five clinical signs in the evaluation of meniscal pathology. Arthorscopy 1989; 5(3): 184-6.

12. Calis M, Akgun K, Birtane M, Karacan I, Calis H, Tuzun F. Diagnostic values of clinical tests in subacromial impingement syndrome. Ann Rheum Dis 2000; 59 (1): 44-7. 